\title{
LA LUCHA POR LOS DERECHOS DE LA MUJER AFRICANA, ¿FEMINISMO? ${ }^{1}$
}

\author{
María Cristina Gallardo Caparrós ${ }^{2}$
}

\begin{abstract}
La lucha por los derechos de la mujer africana, ifeminismo?
Resumen: Al plantearnos la forma en que la lucha por los derechos de las mujeres se ha desarrollado en el contexto africano encontramos, a grandes rasgos, dos posturas principales: aceptar el término feminismo adaptándolo a la realidad africana o rechazarlo por completo para crear nuevas teorías con nombres diferentes. A la hora de abordar el rechazo -parcial o total-que desde algunos ámbitos existe hacia el término feminismo debemos, por un lado, conocer las alternativas propuestas para denominar sus planteamientos teóricos y por otro, reflexionar sobre las motivaciones que han conducido a ese rechazo.

Palabras clave: África, feminismos, womanisms, género, identidad
\end{abstract}

\section{African Women's Fight for their Rights - Feminism?}

Abstract: When we think about how the struggle for women's rights has been developed in the African context, we can find broadly speaking, two main positions: the acceptance of the term "feminism" -adapted to the African reality-, or a total rejection in favour of new theories with different names. In the case of a denial of the term, either total or partial, two important approaches should be addressed. The firs one in order to know the proposed alternatives to name their theories and the second one in order to reflect upon the motivations that led to this rejection.

Key words: Africa, feminisms, womanisms, gender, identity

\section{Introducción}

A partir de los años sesenta del siglo pasado y continuando el trabajo de Sojourner Truth -mediados del siglo XIX-y de Ida Wells -finales del XIX y principios del XX- ${ }^{3}$,

1 Fecha de recepción: 19/11/2020.

Fecha de aceptación: 15/12/2020.

${ }^{2}$ Profesora Sustituta Interina, Departamento de Filología, Universidad de Almería, Almería, España; $\square$ mgc195@ual.es.

${ }^{3}$ Sojourner Truth en la Convención de los Derechos de la Mujer en Akron de 1852 pronuncia el discurso “Ain’t I a Woman?" considerado el texto fundacional del feminismo negro. Ida Wells se dedicó a la denuncia de la discriminación por causa de raza y de sexo tanto desde el ámbito académico como desde el activismo y fue una de las fundadoras de la National Associtation for the Advancement of Colored People y escribió The Red Record (1895). 
comienza a surgir en Estados Unidos un movimiento feminista que se distancia de algunos de los presupuestos del feminismo desarrollado en Occidente. Las reivindicaciones se basan principalmente en que este movimiento se ha configurado y ha evolucionado teniendo en cuenta únicamente la situación de la mujer blanca y occidental, obviando las realidades de otras mujeres que no responden a este patrón identitario.

El interés por incluir dentro del movimiento feminista las especificidades de la mujer de ascendencia africana, se sitúa en Estados Unidos y en el ámbito académico (Pérez 2012: 50). Las reivindicaciones giran en torno a la forma en la que el contexto de la mujer negra y de la sociedad africana ha sido ignorado. Esta omisión de la situación y las características de gran parte de las mujeres del mundo supone -como diría posteriormente Chandra Talpade Mohanty- la creación de una imagen homogeneizada de la mujer negra, "the average third-world woman" (Mohanty 1988: 62), una especie de personaje configurado como una víctima dentro de un grupo homogéneo y juzgado según -y ajustado a- la óptica y los valores de Occidente.

Cuando nos proponemos profundizar en las características y especificidades obviadas por la tradición feminista, resulta especialmente ilustrativa la exposición de la antropóloga Filomina Steady. Entre sus múltiples publicaciones, dedicadas en su mayoría a profundizar en la forma en que la historia ha tratado a la comunidad africana y en concreto a la mujer, nos detendremos en The Black Woman Cross-culturally (1981). En el ensayo que abre esta antología, presenta cuatro realidades de las mujeres africanas que difieren de las de las mujeres occidentales y cuya influencia estima fundamental a la hora de plantearse el feminismo.

Steady aborda en primer lugar la autonomía y la cooperación. A lo largo de la historia, la mujer africana ha gozado de cierta importancia y autonomía en la esfera 
pública, incluso en las sociedades patrilineales ${ }^{4}$, situación que paradójicamente se deriva en gran medida de su papel como madre ${ }^{5}$ (Steady 1981: 29). La segunda especificidad, se refiere a la clásica y recurrente identificación de lo femenino con la naturaleza y lo masculino con la cultura ${ }^{6}$ suponiendo una posición dominante de lo masculino sobre lo femenino. En la tradición y la sociedad africanas, la naturaleza -y lo natural- impera sobre la cultura -tecnología, biología-. Es más, Steady expone cómo, en cierto sentido, se considera que esos avances de la cultura no solo propician el agotamiento de los recursos naturales, sino que además perjudican a las mujeres facilitando la explotación del cuerpo femenino y la perpetuación de una dinámica sexista ya interiorizada en Occidente (Steady 1981: 31-32). La tercera cuestión a tener en cuenta es el empleo del humor y del ridículo para relajar la tensión entre hombres y mujeres, incluso como parte de los rituales -sátiras rituales-(1981: 33-34). Por último, y relacionado parcialmente con ese papel fundamental que juega la maternidad en la sociedad africana, el concepto de familia es mucho más amplio y sólido que en Occidente, los niños son un valor fundamental independientemente de su $\operatorname{sexo}^{7}$ y la figura de la madre ocupa también un lugar privilegiado, de modo que, las relaciones de dominancia o poder que se establecen en el -amplio- núcleo familiar, no pueden identificarse como idénticas a las de Occidente (1981:33).

Con la definición de estas especificidades, Steady no solo demuestra la relevancia de tomarlas en consideración, va un paso más allá y pone en cuestión la supuesta

\footnotetext{
4 "With regard to societies organized on the basis of patrilineal descent, [...] even in this societies there is often a paradox: male dominance on the one hand conflicts with the structural significance odd women as "mothers" on the other. As a result, even women in male-dominated societies have a certain degree of autonomy" (Steady 1981: 29).

${ }^{5}$ La relevancia y la forma de concebir la maternidad se abordará más adelante.

${ }^{6}$ Steady toma el concepto de cultura como próximo al concepto de tecnología, en cuanto a la forma en la que la tecnología destruye lo natural en nombre de la cultura (Steady 1981:31).

${ }^{7}$ En las definiciones Steady, tanto niños como niñas, son vistos como continuidad, unión entre vida y muerte y apoyo a los mayores al final de sus vidas (1981:33).
} 
superioridad de la mujer occidental sobre la mujer africana calificando a esta última como la feminista original: "The majority of the black women in Africa and in the diaspora have developed these characteristics, though not always by choice. [...] it can therefore, be stated with much justification that the black women is to a large extent the original feminist" (Steady 1981: 35-36).

Estos y otros elementos contextuales que diferencian las sociedades entre sí y, por tanto, las realidades de las mujeres han sido abordados en no pocas ocasiones a partir de finales del siglo XX poniendo de relieve que, pese a la importancia de tenerlos en cuenta para plantear la lucha feminista, han sido frecuentemente borrados de sus planteamientos y sus textos teóricos. Este es uno de los puntos de partida de los célebres trabajos de Alice Walker $^{8}$, bell hook ${ }^{9}$ o de The Combahee River Collective ${ }^{10}$.

\section{Aceptación de un feminismo con condiciones}

Los trabajos adscritos al movimiento feminista y que reclaman la consideración de las especificidades de la mujer africana y el reconocimiento por parte del feminismo occidental de haber discriminado a una parte importante de las mujeres, han sido numerosos. Hazel Caraby se apoya en los conceptos de history y herstory ${ }^{11}$ para explicar

\footnotetext{
${ }^{8}$ Alice Walker formulará las bases del Womanism con la idea de abarcar las realidades de todas las mujeres, especialmente de las mujeres negras que han sido ignoradas por la teoría feminista occidental.

${ }^{9}$ bell hook retoma en Ain't I a Woman? Black Women and Feminism (1981) la pregunta formulada más de un siglo antes por Sojourner Truth, para cuestionarse la forma en que el feminismo blanco está borrando e ignorando la experiencia de la mujer negra.

${ }^{10}$ Organización que nace a finales de la década de los 70 del siglo pasado. The Combahee River Collective Statement (1977) se ha convertido en uno de los textos de referencia del feminismo negro.

${ }^{11}$ Término acuñado por el feminismo negro de los años sesenta a partir de los pronombres her/his -su de ella y su de él en inglés-. Mercedes Jarbado en Feminismos negros, una antología decide mantener el original porque considera que no hay traducción posible en español que conserve la potencia del juego de palabras (2012: 210).
} 
su punto de vista. Antes de aplicar los presupuestos del feminismo blanco a la experiencia de las mujeres negras es necesario reconfigurarlos. No se trata solamente de considerar las especificidades de las que hablaba Steady, sino de entender además que, en una realidad donde la discriminación por género se cruza con otras como la raza, hay que tener en cuenta que esa intersección transforma los conceptos sobre los que se fundamenta la lucha feminista (Caraby 1982: 212).

La homogeneización derivada de ignorar la realidad propia de las mujeres no occidentales o no occidentalizadas es uno de los conceptos en los que profundiza Chandra Talpade Mohanty cuando habla de "the average third-world woman". Esta profesora de origen hindú analiza esa imagen homogénea que occidente ha creado de la mujer oprimida del tercer mundo como ignorante, sin educación y anclada en los valores de la tradición y la enfrenta a la forma en que la mujer occidental se presenta a sí misma: liberada y libre en sus decisiones, moderna y con control sobre su cuerpo:

\begin{abstract}
A homogeneous notion of the oppression of women as a group is assumed, [...]. This average third-world woman, leads an essentially truncated life based on her feminine gender (read : sexually constrained) and being 'third world (read : ignorant, poor, uneducated, tradition-bound, religious, domesticated, family-oriented, victimized, etc.).This, I suggest, in contrast to the (implicit) self-representation of western women as educated, modern, as having control over their own bodies and sexualities, and the 'freedom' to make their own decision. (Mohanty 1988: 62)
\end{abstract}

Esta representación desde Occidente como la víctima -la otra-, crea una relación de dependencia en la que la mujer del tercer mundo se define a partir de los rasgos de la mujer occidental y existe dependiendo de ella. En este sentido, podríamos establecer cierta similitud con la relación de dependencia planteada por Sedgwick ${ }^{12}$ al hablar de las

\footnotetext{
${ }^{12}$ Sedgwick en Epistemología del armario (1998) se centra al hablar de los términos A y B en la oposición heterosexualidad/homosexualidad y los emplea para deconstruir el binarismo basándose en la incoherencia de este tipo de oposiciones.
} 
oposiciones binarias en las que el término B existe a partir del término A y viceversa, pero donde los roles de sujeto-objeto permanecen inalterables y definidos desde Occidente (Sedgwick 1998: 20).

Intentando huir de esa homogeneización que no toma en consideración las distintas realidades de las mujeres y las distintas formas de opresión, una de las soluciones propuestas es la matriz de dominación de Patricia Hill Collins y para cuya definición se apoya en la interseccionalidad ${ }^{13}$ como concepto que recoge la interrelación de factores discriminatorios que contribuyen a la opresión del sujeto:

Intersectionality refers to particular forms of intersecting oppressions, [...] Intersectional paradigms remind us that oppression cannot be reduced to one fundamental type, and that oppressions work together in producing injustice. In contrast, the matrix of domination refers to how these intersecting oppressions are actually organized. (Collins 2000: 18)

Partiendo del razonamiento de Collins, la matriz de dominación sería un procedimiento para definir y entender cómo se estructura la intersección de opresiones, ayudando a producir diferentes matrices que recogen una configuración de dominaciones distinta para cada realidad y, derivado de ello, una forma de lucha también distinta en cada caso (Collins 2000: 245). Ama Ata Aidoo considera que las y $\operatorname{los}^{14}$ africanos deben tomar las riendas de su propia lucha (Aidoo 1996: 169) -apropiándonos de los términos de Spivak, el subalterno debe hablar por sí mismo-. Frente a otras posiciones que repasaremos en breve, esta lucha debe emprenderse desde y dentro del movimiento

\footnotetext{
${ }^{13}$ Sobre este concepto versan entre otros los trabajos de Kimberlé Crenshaw, Patricia Hill Collins, Ange Marie Hancock o girando en torno al concepto sin emplearlo directamente Audre Lorde, bell hook, Chimamanda Ngozi Adichie.

${ }^{14}$ Cuando se aborda la cuestión de la lucha por los derechos de las mujeres desde el contexto africano, se rechaza con frecuencia la concepción del hombre como el enemigo y se considera que el hombre debe luchar junto a la mujer por la igualdad.
} 
feminista: "Feminism is an essential tool in women's struggles everywhere, and that includes African women. Every woman, as well as every man, should be all be feminist" (1996:164).

El debate sobre si el contexto africano debe abrazar o no el feminismo, y en ese caso de qué forma, continúa en el siglo XXI. Chimamanda Ngozi Adichie, con su We should all be feminist ${ }^{15}$, está manifiestamente de acuerdo con Aidoo cuando nos dice que el feminismo es una herramienta esencial y plantea varias cuestiones interesantes. Una de ellas es que ese rechazo se produce tanto desde la comunidad académica como desde la sociedad en general. Las citas siguientes reflejan esa concepción del movimiento como una traición a la tradición y a las raíces africanas o incluso un ataque:

Okoloma era uno de mis mejores amigos de infancia. [...] Yo tenía unos catorce años. [...] Okoloma me miró y me dijo:

$-i$ Sabes que eres una feminista?

No era un cumplido. Me di cuenta por el tono en que lo dijo, el mismo tono con que alguien te podía decir: "Tú apoyas el terrorismo". (Adichie 2019:6)

En 2003 escribí una novela titulada La flor púrpura [...] Por aquella época una académica, una mujer nigeriana, me dijo que el feminismo no era nuestra cultura, que el feminismo era antiafricano, y que yo solo me consideraba feminista porque estaba influida por los libros occidentales. (Adichie 2019: 7)

Aunque en la obra de Adichie no encontramos ninguna referencia directa a la interseccionalidad, los análisis sobre sus textos sí relacionan sus propuestas con el feminismo interseccional y con muchas de las ideas que bell hook introdujo en Feminism is For Everybody: Passionate Politics (2000); ambas producciones se consideran intentos

\footnotetext{
${ }^{15}$ Título de la charla TED de Chimamanda Ngozi Adichie (diciembre de 2012) en TEDxEuston y que posteriormente convertiría en un ensayo publicado en 2014 bajo el mismo título.
} 
de contentar a todo el mundo obviando los límites y peligros de este enfoque (Paniagua 2019: 136).

\section{El rechazo de un feminismo africano a la occidental}

Estas posturas críticas hacia el feminismo occidental no están exentas, a su vez, de críticas y detractores. En concordancia con las apreciaciones de Susan Arndt cuando nos dice que muchas de estas propuestas -sobre todo las desarrolladas entre los años 60 y los años 80- están centradas en la mujer afroamericana (Arndt 2002: 53), podemos apreciar la aparición de una especie de feminismo desde la diáspora -no para la diáspora- que, pese a las buenas intenciones iniciales, ejercerá sobre las mujeres del continente africano una exclusión similar a la reclamada al movimiento Sufragista o a Betty Friedan ${ }^{16}$.

Desde algunos sectores que luchan por los derechos de las mujeres, se da una ruptura aun mayor y surgen académicas, escritoras y activistas que no solo rechazan la parte de los presupuestos feministas que las homogeniza y las silencia, deciden ir un paso más allá apartándose drásticamente del movimiento feminista y negándose a ser identificadas con él. La escritora camerunesa Leonora Miano ${ }^{17}$ es ejemplo de uno de estos casos. En una entrevista concedida a la revista Les Inrockuptibles en 2019, declaraba no sentirse identificada con este movimiento por considerar que no se ha conseguido una verdadera sororidad en su seno y que se desarrolla principalmente desde una perspectiva

\footnotetext{
${ }^{16}$ Se ha referido con frecuencia que el movimiento sufragista -finales del siglo XIX hasta mediados del XX- y Betty Friedan -a principios de los 60- plantearon sus reivindicaciones desde la generalización y la consideración de los valores y de la situación de la mujer blanca occidental como únicos y universales. ${ }^{17}$ Reconocida autora camerunesa, aborda en sus relatos la problemática de las mujeres junto a otros temas como los conflictos del África subsahariana, el pasado colonial o el racismo.
} 
occidental que no tiene en cuenta ni la historia, ni las luchas, ni las grandes figuras feministas africanas:

Je ne suis pas féministe, même si je suis une femme qui veut absolument être libre et faire ce qu'elle veut dans la vie [...] d'abord parce que je ne pense pas qu'il faille que nous nous soumettions à la domination épistémologique de l'occident [...] en Afrique nous avons notre historie, nos luttes et nos grandes figures féminines [...] nous ne sommes pas obligés d'appeler ça du féminisme. On peut lui trouver un autre nom. C'est donc ma première [...] La deuxième, c'est que je ne suis pas entrée et je ne vis pas dans un monde où les femmes aiment les autres femmes. [...] c'est ça : ne pas avoir permis aux femmes d'aimer être des femmes et donc d'aimer a priori les autres femmes. Quand le féminisme aura résolu ça, je serai peut-être féministe. $\left(\right.$ Miano 2019) ${ }^{18}$

Las escritoras senegalesas Khadi Hane y Aminata Sow Fall ${ }^{19}$ también rechazan su identificación como feministas haciendo referencia a la fama que el movimiento tiene en el contexto africano. "Je ne suis pas de ces femmes africaines qui rejettent de leur culture tout en bloc pour faire la place au féminisme tel qu'il est véhiculé en occident. Je ne suis pas féministe, mais humaniste" (Hane 2011) ${ }^{20}$. A partir de estas palabras de Hane, vemos cómo el hecho de identificarse con el feminismo se relaciona con abrazar la cultura occidental traicionando las raíces y a la cultura africana. Precisamente en esa línea de huir de la etiqueta de "traidora de raíces" se enmarca también Sow Fall cuando declara "Je

\footnotetext{
${ }^{18}$ Miano, Leonora. (2019, 13 de agosto) 'Rencontre avec Léonora Miano : ' Comment pacifier à l'intérieur de soi la relation avec l'ancien colon ? “ “. Entravistada por Léonard Billot. Les Inrockuptibles, 1238. https://www.lesinrocks.com/2019/08/13/livres/livres/rencontre-avec-leonora-miona-je-nhabite-pas-lemonde-en-me-sentant-victime-de-lhistoire/. [12/12/2020]

${ }^{19}$ Autoras del panorama francófono (de origen senegalés) que, por la forma de tratar la situación de las mujeres y los tintes de crítica a la ideología patriarcal, son consideradas en muchas ocasiones feministas o defensoras de los derechos de la mujer.

${ }^{20}$ Hane, Khadi. (2011, 15 de septiembre) "Interview de Khadi Hane à propos Des fourmis dans la bouche". Chez Gangoueus. http://gangoueus.blogspot.com/2011/09/interview-de-khadi-hanepropos-des.html. [27/06/2020].
} 
n'ai pas été influencée par le courant du féminisme. Pour la bonne raison que moi, je ne suis pas féministe dans le sens où l'entendent les gens" (Sow Fall 1986 :294) ${ }^{21}$.

Un último ejemplo de esta postura -más conocida que las anteriores en el panorama académico internacional- es Buchi Emecheta quien, aun reconociendo cierta afinidad entre su modo de vida y los presupuestos feministas, rechaza la etiqueta y rechaza ser identificada con la mujer europea: "I will not be called a feminist here, because it is European. It is as simple as that. I just resent that. Otherwise, if you look at everything I do, it is what the feminists do, too; but it is just that it comes from Europe, or European women, and I don't like being defined by them" (Emecheta 1985) 22 .

Estas declaraciones son ejemplos de mujeres que, aun dedicando su carrera a la reivindicación de la independencia y la libertad para las mujeres, rechazan el feminismo huyendo de los matices de occidentalización y de reproducción de las prácticas coloniales que tiene el término en el contexto africano. Esta huida ha dado lugar a diversas corrientes de pensamiento que, no solo ponen de manifiesto esa ruptura con lo occidental, sino que se renombran ${ }^{23}$ lejos del feminismo o de los feminismos para marcar esa distancia.

\section{Renombrarse para distanciarse}

La cuestión de la terminología apropiada para hablar sobre la situación de la mujer y la lucha por sus derechos en el continente africano ha sido largamente debatida.

\footnotetext{
${ }^{21}$ Entrevista con Aminata Sow Fall "Interview d'Aminata Sow Fall” en Milolo, K. 1986. L'image de la femme chez les romancières de l'Afrique noire francophone. Editions universitaires. (292-295)

${ }^{22}$ Entrevista a Buchi Emecheta en Umeh, Davidson, and Marie Umeh. 1985. "An Interview with Buchi Emecheta." Ba Shiru 12. 2: 19-25.

${ }^{23}$ La relevancia de encontrar ese nuevo concepto viene de la importancia que tiene en la cultura africana que cada nombre tenga un significado, porque otorga poder a la realidad nombrada - "naming is power [...] In my culture every name has a meaning"-(Arndt 2000: 721).
} 
Cleonora Hudson-Weems -creadora del African Womanism y muy crítica con el movimiento feminista como veremos a continuación-considera que las defensoras de los derechos de la mujer en el contexto africano se han unido al movimiento feminista ante la falta de teorías propias más adecuadas a su realidad (Arndt 2002: 40).

En este sentido, Mohanty plantea que el trabajo a realizar debe emprenderse a partir de la deconstrucción de los fundamentos y principios del feminismo de Occidente -tarea en la que se centra en Under Western Eyes (1991)- para así poder (re)construir alternativas y teorías propias (Mohanty 1991: 51-52).

Es en esa tarea de reconstrucción en la que se centran las formulaciones que vamos a ver a continuación. La mayoría de ellas, ${ }^{24}$ además de proceder a esa reformulación y (re)construcción de bases se afanan en renombrarse y así proyectarse a la sociedad a modo de alternativas alejadas de las connotaciones negativas del término feminismo. Como sucediera en Occidente, se van a desarrollar distintas teorías en las que, partiendo de la preocupación -compartida con el feminismo- por identificar y superar los mecanismos de opresión (Arndt 2002: 63), las diferencias van a radicar principalmente en cómo se entienden ciertos conceptos básicos y en el alcance de realidades que no existen en Occidente $-\mathrm{o}$ que se entienden $\mathrm{y}$ se viven de manera radicalmente distinta-: la importancia concedida a maternidad, la visión de la poligamia, la participación y el papel del hombre en la lucha por los derechos de las mujeres o la homosexualidad femenina son las cuestiones tratadas con más frecuencia.

\footnotetext{
24 Algunas de estas propuestas se autodefinen conservando la palabra feminismo. Es el caso del Negrofeminism de Obioma Nnaemeka.
} 


\title{
5. El Womanism de Alice Walker
}

El Womanism de la afroamericana Alice Walker es probablemente la teoría más cercana al feminismo y una de las más abordadas en los textos teóricos. El concepto nace a principios de los ochenta a partir de la obra de Alice Walker ${ }^{25}$, atendiendo a las especificidades que se han obviado en la teoría feminista occidental sobre la mujer africana del continente y la de la diáspora. Walker, en In Search of Our Mothers' Gardens (1983) ofrece una definición de la mujer Womanist que tomaremos a modo de definición de esta corriente, y que explica la forma en que se relaciona con el movimiento feminista ${ }^{26}$ :

\begin{abstract}
A black feminist or feminist of colour [...] A woman who loves other women, sexually and/or nonsexually. Appreciates and prefers women's culture, women's emotional flexibility (values tears as natural counterbalance of laughter), and women's strength. Sometimes loves individual men, sexually and/or nonsexually. [...] Loves music. Loves dance. Loves the moon. Loves the Spirit. Loves love and food and roundness. Loves struggle. Loves the Folk. Loves herself. Regardless. [...] Womanist is to feminist as purple to lavender. (Walker 1983: 7)
\end{abstract}

En cuanto al papel que juega el hombre dentro del Womanism y partiendo de la idea de comunidad $^{27}$, aunque solo las mujeres pueden ser Womanists, sí se considera que debe

\footnotetext{
${ }^{25}$ La publicación de In Search of our mothers' garden tiene lugar el 1983 aunque algunos autores como Isabel Pascua Febles en El Womanism, visión de la mujer africana en la literatura postcolonial (2017) sitúan el inicio del Womanism en 1979 con la publicación del relato corto de Walker Coming Apart (1979). Por otra parte, el término Womanism ha sido traducido en algunos textos como mujerismo, aunque la tendencia general es mantener el término en inglés.

${ }^{26}$ La última frase de esta cita "Womanist is to feminist as purple to lavender" es una de las más recurrentes cuando se aborda la definición del Womanism.

${ }^{27}$ Teniendo en cuenta la importancia que el estudio de la obra de las creadoras africanas ha ido tomando en occidente, y la concepción en el contexto africano de que toda la comunidad debe participar en la lucha por los derechos de las mujeres y su representación en el ámbito literario; encontramos, el artículo que María Carreño López dedica a la mozambiqueña Noémia de Sousa. La autora del artículo destaca que la poética de la mozambiqueña posee "un cariz colectivo" que la lleva a "rechazar los intentos de forzar la poesía de Noémia en moldes feministas" (Carreño 2013: 5).
} 
ser consciente de la discriminación sufrida por las mujeres porque, aun tratándose de luchas distintas, hombres y mujeres deben aunar esfuerzos en el proceso de liberación de la dominación occidental (Pérez 2012: 57).

La figura de la madre, gozando de una importancia mayor que en la óptica occidental, no se plantea esencial para el desarrollo de la vida de las mujeres como ocurre en otras perspectivas en las que se convierte en casi en una obligación. Esta cuestión será una de las más criticadas del planteamiento walkeriano junto con la aceptación de la homosexualidad femenina. ${ }^{28}$

\section{El African Womanism de Chikwenye Okonjo Ogunyemi}

La forma en que la óptica occidental percibe las costumbres y tradiciones africanas presentes en la vida de una mujer tiene para Ogunyemi tintes voyeristas porque ignora muchos aspectos relevantes para concentrarse en aquellos rasgos que pueden resultar más exóticos o polémicos, de manera que se las convierte en objetos de la cultura occidental y se crea una distancia que impide la colaboración y el entendimiento entre las mujeres africanas y las occidentales (Arndt 2000:724).

Aunque afín al Womanism de Walker en ciertos aspectos, las diferencias entre las africanas del continente y las afroamericanas exigen una teoría propia para el primer grupo que acoja las inquietudes de las mujeres no identificadas con los planteamientos walkerianos, en palabras de la propia Ogunyemi, "there is a need to define African

\footnotetext{
${ }^{28}$ Representantes de otras corrientes de pensamiento feministas africanas han puesto de relieve que este Womanism omite la singularidad propia del continente africano -Chikwnye Okonjo Ogunyemi- o que está lleno de contradicciones y cargado de tintes lésbicos -Catherine Obianuju Acholonu- (Pérez 2012: 57-59). 
womanism. It is necessary to reiterate that the womanist praxis in Africa has never totally identified with all the original Walkerian precepts" (Ogunyemi 1996: 133).

Para la definición de las características principales del African Womanism, nos remitiremos a las declaraciones de la propia Ogunyemi en una entrevista con Susan Arndt. En la introducción de la entrevista, Anrndt pone de relieve que dos de las diferencias más relevantes entre Walker y Ogunyemi radican en sus aproximaciones a la maternidad y a la homosexualidad femenina. A la primera de estas cuestiones, no se le concede en el Womanism la importancia que merece llegando, en cambio, a verse en el African Womanism casi como un deber. En cuanto a la segunda cuestión -la homosexualidad femenina- tanto para Ogunyemi como para el resto de propuestas, es un tema que prefieren no abordar y, en las ocasiones en las que se menciona, adquiere un halo de desaprobación porque para la cultura africana, atenta contra el matrimonio y contra la familia. (Arndt 2000: 712)

En lo que se refiere a la realidad de la poligamia, aunque a lo largo de esta entrevista Ogunyemi denuncia la opresión que ejercen los maridos sobre sus esposas y unas coesposas sobre otras, también comenta la existencia de un punto de vista que extraña a menudo en Occidente; el rechazo de la monogamia en pro de la poligamia. Esta aceptación se produce a partir de la deconstrucción del concepto para convertirlo en un medio de liberación e independencia en el que las cargas que supone el matrimonio y la familia son compartidas (Arndt 2000: 714-716). Ogunyemi se apoya en este ejemplo y en el drástico rechazo de este planteamiento por parte del feminismo para ilustrar la necesidad de construir una definición y una alternativa propias de lucha por los derechos de las mujeres, que para ella se materializa en el African Womanism. 
Este African Womanism cuenta entre sus objetivos con la necesidad de independencia de la mujer sobre todo en lo que a medios económicos y materiales se refiere, y esa independencia debe no solo ser real sino también ser percibida por sus congéneres. Se busca establecer una relación de respeto entre unos y otros, meta a la que la autosuficiencia contribuye en gran medida (Arndt 2000: 717-718). No obstante, esta búsqueda de libertad y autonomía no sitúa a los hombres enfrente sino al lado, la sensibilización del hombre en lo que se refiere a los derechos de la mujer es indispensable para que se una a la lucha por el cambio y en este sentido el empleo del término “feminismo" provocaría el efecto contrario:

I think one of the ways in which they can become exposed to the idea of a change is not to use names like "feminism." This name would alienate them from our ideas. So you call it something else because it is something different. [...] If you begin to name your own activity yourself, there is power in that naming. And when they see that there is power in the naming and also that you do not take them as enemies, men agree to be a part of the change [...] Whereas, if you just take the women alone and deal only with the women, then you are going to come back to the men, who have not changed at all. (Arndt 2000: 717)

\section{El Africana Womanism de Cleonora Hudson-Weems}

Frente al African Womanism que nace dirigido hacia las africanas del continente, el Africana Womanism de Hudson-Weems pretende ser punto de encuentro de todas las mujeres de ascendencia africana ${ }^{29}$. La distancia con el feminismo se justifica por su intención de enmarcar la historia y la realidad de todas las mujeres bajo la óptica de las mujeres blancas de forma que se hace necesario un movimiento que parta y se centre en las experiencias, luchas, necesidades y deseos de las africanas, en este sentido "Africana Womanism and its agenda are unique and separate from both White feminism and Black

\footnotetext{
${ }^{29}$ El detalle de las dieciocho características de una Africana Womanist puede consultarse en HudsonWeems 1993 Africana Womanism: Reclaming ourselves (55-73).
} 
feminism, and moreover, to the ex- tent of naming in particular, Africana Womanism differs from African feminism" (Hudson-Weems 1993: 24).

La exaltación de la especificidad africana es llevada un paso más allá cuando Hudson-Weems afirma que Sojourner Truth o Ida B. Wells deben ser consideradas Africana woman frente a la visión de la historia feminista que las califica de Pre-feminist. Es más, acusa al feminismo blanco de haber tomado la vida y obra de las Africana heroines en un intento de legitimar su movimiento de mujeres como el único y real (Hudson-Weems 1993: 22).

Siguiendo las directrices del Africana Womanism, la propuesta de Walker presta excesiva atención a las cuestiones de género en detrimento de las igualmente importantes cuestiones de raza o clase. "Race and class biases are the key issues for non-Whites and must be resolved even before gender issues if there is any hope for human survival" (Hudson-Weems 1993: 41), es decir, la lucha no puede limitarse a los derechos de la mujer sino que debe extenderse al resto de discriminaciones que sufre la población porque la liberación de la opresión de género no podrá alcanzarse hasta que no se alcance la liberación como africana (1993: 58-59).

\section{El Motherism de Catherine Obianuju Acholonu}

De la mano de esta nigeriana nos llega una perspectiva que no acepta los principios que se asocian tradicionalmente al movimiento feminista por promover valores contrarios al modo de vida africano; por ser -según la propia Acholonu- "anti-mother, anti-child, anti-nature and anti-culture” (Acholonu 1995: 82). Es decir, las prácticas feministas incluidas las africanas- apoyan actitudes como la exclusión del hombre y la ruptura de 
las bases de las relaciones entre hombres y mujeres -mechanical equity-, y en cambio la relaciones de género deberían regirse por la complementariedad-complementarity, es decir, "Equality is confrontational and self destructive; complementarity is diplomatic, mutually supportive, and dynamic" (Acholonu 1995: 107).

El o la motherist, basa su vida en el servicio a los demás y no ejerce ningún tipo de opresión ni discriminación ya sea de género, raza o religión. Los principios que rigen su vida y su escala de valores son la maternidad, la naturaleza y la crianza -motherhood, nature and nurture-:

\begin{abstract}
A man or a woman [...] a builder, a healer, not a destroyer, a co-creator with God, a lover of the child who loves and respects all men and women irrespective of colour, race, ethnicity, cultures and religions [...] ever poised to question the status quo, ever ready to promote reforms, ever ready to make personal sacrifices for the good of others like any mother would, for no matter his/her age or sex the motherist is essentially a mother. (Acholonu 1995: 112)
\end{abstract}

La naturaleza se presenta en grado de importancia a la altura de la figura de la madre. Partiendo de la relevancia de estos dos conceptos, la mujer rural se convierte en una figura digna de admiración y veneración puesto que representa todas las cualidades de la madre tierra, "It is life; it is love; it is spirituality; it is the creative, nurturing power of God, present in the female principle" (Acholonu 1995: 119) y sería su encarnación humana.

\title{
9. El Stiwanism de Molara Ogundipe-Leslie
}

El movimiento Social Transformation Including Woman in Africa-STIWAN-, 
iniciado por la escritora y activista nigeriana Molara Ogundipe-Leslie, nace con la intención de desmarcarse de la imagen del feminismo tradicional y de promover la participación activa de las mujeres como un actor más en la necesaria transformación social de África (Ogundipe-Leslie 1994: 230). Aunque evidentemente el Stiwanism también se aleja del feminismo occidental, no se distancia tanto como los Womanisms de Ogunyemi y de Hudson-Weems o el Mothersim - aunque sin acercarse a la visión de Alice Walker- y acepta una especie de feminismo con condiciones, un feminismo alejado de los extremos, de la división y de la discriminación:

1.Feminism is not a cry for any one kind of sexual orientation and I am not Homophobic or heterosexist. [...] 2. Feminism is not the reversal of gender roles, "gender being defined simply as socially constructed identities and roles. [...] 3. Feminism is not penis envy or gender envy; wanting to be a man as they like to say to us[...] 5. Feminism is not "dividing the genders" [...]6. It is not patriotism of Western women's rhetoric [...] 7. It is not opposed to African culture and heritage [...] 8. Feminism is not a choice between extreme patriarchy on the one hand or hateful separatism from men on the other. (Ogundipe-Leslie 1994: 222)

No obstante, la creación de este nuevo término viene motivada por el deseo de evitar el rechazo del continente africano, se opta por evitar la palabra feminismo "to bypass the combative discourses that ensue whenever one raises the issue of feminism in Africa" (Ogundipe-Leslie 1994: 229). Es una propuesta adaptada exclusivamente al contexto africano que no recoge las inquietudes ni de la mujer blanca ni de la afroamericana y que pretende luchar directamente contra la discriminación de género como objetivo primordial $^{30}$. Se considera que obviar los conflictos que pueden surgir entre esta y otras

\footnotetext{
${ }^{30}$ En este sentido se establece una de las principales diferencias con otras teorías como el Motherism o el Africana Womanism en las que esta cuestión se sitúa al mismo nivel que otras formas de opresión o incluso en un segundo plano.
} 
formas de opresión "is to be either romantic, hypocritical or dishonest, or perhaps just unintelligent" (1994: 207).

Otra de las cuestiones en las que el Stiwanism se sitúa a medio camino entre la concepción africana del feminismo occidental y la mayoría de las propuestas que ya hemos comentado, es en el papel que juegan los hombres dentro de este escenario. No se ve en la figura masculina un enemigo en sí, pero se puntualiza que puede llegar a serlo cuando adopta una postura de bloqueo ante los cambios históricos y sociales (OgundipeLeslie 1994: 82). La base para esos cambios está en la superación de seis obstáculos, montañas en términos de Ogundipe-Leslie, comenzando por la opresión exterior y terminando en el interior de la propia mujer que, tras tanto tiempo bajo la jerarquía de los roles de género se encuentra anclada en un complejo de inferioridad que le impide, precisamente, reconocer su propia situación:

One migth say that the Africa woman has six mountains on her back: one is oppression from outside (colonialism and neo-colonialism), the second is from traditional structures, feudal, slave-based, communal etc., the third is her backwardness (neo-colonialism); the fourth is man; the fifth is her color, her race; and the sixth is herself. (1994: 28)

\section{La Misovire de Werewere Liking}

La escritora camerunesa Werewere Liking crea este término a partir de la ficción literaria en su novela Elle sera de jaspe et de corail: Journal d'une Misovire (1983), no obstante, y es por eso que la tratamos aquí, dentro de la historia se crea como una perspectiva real de concebir las relaciones entre hombres y mujeres. Para ello Liking 
configura intencionadamente un neologismo que recoja su voluntad de alejarse del término misántropo y se acerque al concepto de misógino; “Certains m'ont critiquée en disant que ce mot n'éxistait pas. Ils auraient préféré 'misandre' ou je ne sais quel autre terme. Je préférais un terme plus proche de misogyne” (Liking $1985: 18^{31}$ ).

La esencia de este concepto propuesto por Liking nace del personaje principal de su obra que sufre una de las peores situaciones a las que se puede enfrentar una Misovire: no encontrar a un hombre digno de admiración. Se trata de "Une femme incroyable, fragile dans sa chair [...] à l'affût du mot mot-force qui formulera et manifestera son rêve, un rêve chaud dans un corps qu'elle craint n'être que de 'la viande' ... Une misovire quoi !" (Liking 1983: 8-9).

Uno de los acercamientos al concepto de Liking que considera que se trata de una forma nada convencional de entender las relaciones entre los géneros, es el de la profesora camerunesa Eunice Fonyuy Fom que incluso ve en él la posibilidad de una nueva interpretación de la concepción de humanismo que se aleja de la división entre hombres y mujeres y concibe la sociedad como un todo distanciado de las dinámicas de creación y exclusión del otro:

Liking's remarks are not intended to alienate men from women, but to make them think about gender relationships and reinvent themselves (...) Liking's stance is both appealing and inspiring, for I interpret her definition of misovire as a willingness to make a decisive departure from conventional meaning. In this context, Liking's position reveals an interest not so much in feminism as in a new form of humanismnot a humanism that was distorted by the West at a certain point in history and served as a tool to exclude the "other", but a humanism that tolerates no exclusion, a humanism that is capable of transforming society as a whole. (Fombele 2016: 375376)

\footnotetext{
${ }^{31}$ Entrevista realizada a Werewere Liking por Bernard Magnier. Liking, Werewere. 1985. "A la rencontre de... Werewere Liking. Notre librairie 19: 17-22. https://gallica.bnf.fr/ark:/12148/bpt6k6503139j/ f5.image.r=MISOVIRE. [18/09/2020]
} 


\section{Consideraciones finales}

La creación y definición de estas propuestas nace de la necesidad de alejarse de reivindicaciones que crean una imagen de la mujer africana en lugar de aproximarse a la imagen real. Sin embargo, hay ciertas voces que consideran que esta autodefinición se realiza en la mayoría de los casos a partir -y en dependencia de- la mujer occidental. En consecuencia y aunque se consigue superar parte del problema inicial planteado conseguir una definición adaptada a diferentes realidades-, no se supera completamente esa dependencia de los conceptos occidentales que sitúan a la mujer africana como la otra, o como decía Sedgwick, esa existencia dependiente. Pinkie Mekgwe estima que la manera en que estas definiciones se realizan sigue, de una forma y otra, situando a Occidente en el centro y victimizando a la mujer africana sin escapar por completo de la “trampa colonial”. En sus propias palabras: "While Africa still defines herself against the West in much the same way as the West has, since the colonial era, constructed Africa as an 'other', Africa is still enmeshed in a 'colonial trap' and has not yet reached selfdefinition and, by implication, total Independence" (Mekgwe 2006: 21-22).

En cualquier caso, estas propuestas deciden desmarcarse de los presupuestos del feminismo occidental de forma radical, apartándose incluso de términos como feminismo negro, feminismo postcolonial o feminismo del tercer mundo ${ }^{32}$; buscan identificarse y ser identificadas fuera del movimiento. Se reclama la comprensión y el entendimiento de que la óptica occidental no es la única que existe y que por tanto no debe emplearse para analizar y juzgar realidades no occidentales. En este sentido, sería procedente cuestionarse las motivaciones de este deseo. Si nos tornamos hacia algunos ejemplos con

\footnotetext{
32 Nótese que ha existido polémica sobre las fórmulas "feminismo del tercer mundo" y "feminismo postcolonial”. A este respecto puede consultarse: Mohanty 1991:75 y West 1991:IX)
} 
origen en el continente -como el de Ogundipe-Leslie- o en la diáspora -como la propuesta de Walker-, no se trata de un rechazo tajante e íntegro de los principios de todos los feminismos sino de la denominación en sí. Este alejamiento de la palabra "feminismo" se aproxima entonces a una especie de estrategia de marketing que intenta alejarse de las connotaciones negativas del propio término. Partiendo de este hecho, sería interesante plantearse de dónde viene esa imagen tan negativa y extendida. Reflexionar sobre si nace del propio contenido de las ideas del feminismo y de si el movimiento se ha aproximado - o no- a los problemas de las mujeres no occidentales, o surge en cambio de la manera de presentar y transmitir esas ideas, es decir, si parte de la esencia misma del feminismo o -permitiéndonos continuar con la analogía del marketing- de la imagen de marca proyectada.

\section{Referencias Bibliográficas}

Acholonu, Catherine Obianuju. 1995. Motherism: The Afrocentric alternative to feminism. Nigeria: Afa Publications.

Adichie, Chimamanda Nogozi. 2019. Todos deberíamos ser feministas. Literatura Random House.

Aidoo, Ama Ata. 1996. "Literature, feminism and the African woman today". Reconstructing Womanhood, Reconstructing Feminism: Writings on Black Women. Delia Jarrett-Macauley (ed). Londres: Routledge. 156-174.

Arndt, Susan. 2000. "African gender trouble and African womanism: an interview with Chikwenye Ogunyemi and Wanjira Muthoni”. Signs: Journal of women in culture and society 25.3: 709-726.

Arndt, Susan. 2002. The dynamics of African feminism: Defining and classifying Africanfeminist literatures. Trenton: Africa World Press.

Caraby, Hazel. 1981. "White woman listen! Black feminism and the boundaries of "sisterhood"'. Centre for Contemporary Cultural Studies". 2005. EMPIRE 
STRIKES BACK: Race and Racism in 70's Britain. Birmingham: Taylor \& Francis e-Library.

Carreño López, María. 2013. "Noémia de Sousa: la voz histórica de Mozambique. Álabe 7. http//:www.revistaalabe.com. [10/09/2020]

Collins, Patricia Hill. 2000. Black feminist thought: knowledge, consciousness, and the politics of empowerment. New York: Routledge.

Fombele, Eunice Fombele. 2016. "Mythoform and the Misovire Consciousness: A Gender Conceptual Renewal Strategy in Werewere Liking's It Shall Be of Jasper and Coral". Journal of Literature and Art Studies 6.4: 369-379.

Hudson-Weems, Cleonora. 1993. Africana womanism: Reclaiming ourselves. Troy, N.Y.: Bedford Publishing.

Jabardo, Mercedes. 2012. Feminismos negros. Una antología. Madrid: Traficantes de Sueños.

Mekgwe, Pinkie. 2006. "Theorizing African Feminism(s). The 'Colonial' Question". Quest. An African Journal of Philosophy 1.2 : 11-22.

Mohanty, Chandra Talpade. 1988. "Under Western eyes: Feminist scholarship and colonial discourses". Feminist review 30: 61-88. . 1991. "Under Western eyes: Feminist scholarship and colonial discourses".. Third world women and the politics of feminism. C. T. Mohanty, A. Russo \& L. Torres (eds.). Bloomington: Indiana University Press. 51-80.

Ogundipe-Leslie, Molara. 1994. Re-creating ourselves: African women \& critical transformations. Trenton: Africa World Press.

Ogunyemi, Chikwenye Okonjo. 1985. "Womanism: The dynamics of the contemporary black female novel in English". Signs: Journal of women in Culture and Society 11.1: 63-80.

. (1996). Africa wo/man palava: The Nigerian novel by women. Chicago: University of Chicago Press.

Paniagua, Maya Del Puig Zalbidea. 2019. “Por qué todos deberíamos ser feministas? Chimamanda Ngozi Adichie y el feminismo global”. Dossiers feministes 25: 131146.

Pérez Ruiz, Bibian. 2012. Lo lejano y lo bello: feminismos y maternidades africanas a través de su literatura. Madrid: Editorial Fundamentos.

Sedgwick, Eve Kosofsky. 1998. Epistemología del armario. Barcelona: Ediciones de la Tempestad.

Steady, Filomina Chioma. 1981. The Black Women Cross-Culturally. Cambridge: Schenkman Publishing Company.

Walker, Alice. 1983. In Search of Our Mothers' Gardens. San Diego: Harvest. 
Werewere, Liking. 1983. Elle sera de jaspe et de corail: Journal d'une misovire. Paris: L'Harmattan. 